\title{
A comparison of using a smartphone versus a surgical microscope for microsurgical anastomosis in a non-living model
}

\author{
Surut Jianmongkol, Chaiyos Vinitpairot, Navapong Thitiworakarn, Settapon Wattanakamolchai \\ Hand and Reconstructive Unit, Department of Orthopedics, Faculty of Medicine, Khon Kaen University, Khon Kaen, Thailand
}

Background Although they may not replace standard training methods that use surgical microscopes, smartphones equipped with high-resolution screens and high-definition cameras are an attractive alternative for practicing microsurgical skills. They are ubiquitous, simple to operate, and inexpensive. This study compared anastomoses of chicken femoral vessels using a smartphone camera versus a standard operative microscope.

Methods Forty anastomoses of non-living chicken femoral vessels were divided into four groups. A resident and an experienced microsurgeon performed anastomoses of femoral chicken vessels with 8-0 and 10-0 sutures, using a smartphone camera and a surgical microscope. The time to complete the anastomosis and the number of anastomosis errors were compared using the Mann-Whitney $\mathrm{U}$ test.

Results The time taken to perform an anastomosis by the experienced microsurgeon was significantly longer when using the smartphone (median: 32.5 minutes vs. 20 minutes, $\mathrm{P}<0.001$ ). The resident completed the anastomoses with both types of equipment without a significant difference in the operative times. When using a smartphone, the operation times were not significantly different between the resident and the experienced microsurgeon $(P=0.238)$. The resident showed non-significant differences in operation time and the number of errors when using a smartphone or an operative microscope $(P=1.000$ and $P=0.065$, respectively).

Conclusions Microsurgical practice with non-living chicken femoral vessels can be performed with a smartphone, though it can take longer than with an operative microscope for experienced microsurgeons. The resident may also experience frustration and tend to make more anastomosis errors when using a smartphone versus an operative microscope.

Keywords Smartphone / Microsurgery / Blood vessels / Anastomosis, surgical
Correspondence: Chaiyos Vinitpairot Department of Orthopedics, Faculty of Medicine, Khon Kaen University, 123 Mitraphap Road, Khon Kaen 40002, Thailand

Tel: $+66-43348398$

Fax: +66-43364069

E-mail: chaivi@kku.ac.th

Received: June 22, 2021 • Revised: August 31, 2021 • Accepted: September 30, 2021

pISSN: 2234-6163 • elSSN: 2234-6171 • https://doi.org/10.5999/aps.2021.01340 • Arch Plast Surg 2022;49:121-126

\section{INTRODUCTION}

Opportunities for training in microsurgical skills are limited by the high cost of microsurgery training microscopes and limited access to equipment, especially during the coronavirus disease 2019 pandemic era. Modern smartphones have high-resolution screens and high-definition cameras and might be used as an alternative tool by surgical residents and fellows to practice their 
microsurgery skills at home or wherever convenient.

To improve their microsurgery skills, surgical trainees practice using microsurgery training microscopes in a skills lab. These microscopes are expensive instruments to purchase and maintain, and access to the microsurgery skills lab can be difficult. Smartphones and tablet devices have been used in several ways within health care services and training, including surgery [1]. This study describes how a smartphone can be adapted with a simple set-up to be used as a microscope for magnifying the surgical site and thus be an effective instrument for practicing microsurgery.

The first report of using smartphones in microsurgery training was by Kim et al. in 2014 [2]. They used iPhone 5S (Apple Inc., Cupertino, CA, USA) and Galaxy S4 (Samsung, Seoul, Korea) smartphones equipped with high-quality built-in cameras to magnify the operation field. The setting was anastomosis of 2-mm and 4-mm diameter synthetic blood vessels using 8-0 suture material by first-time practitioners. This highlighted the possibility of using smartphones to replace personal loupes or operating microscopes in basic microsurgery training. In 2017 Huotarinen et al. [3] developed a coffee cup and smartphone (CCS) training device to use instead of a microscope. In that study, they compared the performance of first-time trainee surgeons during end-to-end artificial bypass surgery before and after 5 days of training with the CCS device and concluded that their device was efficient, mobile, and easy to set up. Malik et al. [4] reported that home training in microsurgical skills with an iPad was comparable to training with a table-top microscope in the medical student practice program. In 2018, Karakawa et al. [5] compared performing microsurgery using an iPad Pro (Apple Inc.) with an operating microscope. They reported that binocular vision could not be achieved with the iPad Pro. Recently, a study comparing the effectiveness of using a surgical loupe, an operating microscope, and a smartphone in a living model con- cluded that living model blood vessels could not be repaired using a smartphone $[6,7]$. To our knowledge, there is no study comparing the use of a smartphone device or a surgical microscope for microsurgery skill practice in a non-living animal vessel model. The advantage of non-living vessel models, such as those using chicken vessels, is that they are easier to acquire and can be less frustrating to practice on.

The objective of this study was to compare the length of time taken to perform surgery and the error rate in anastomosis of non-living chicken femoral vessels using a smartphone and a surgical microscope. We hypothesized that anastomoses performed using a smartphone would not be different from anastomoses performed using a microscope.

\section{METHODS}

The study design was a prospective comparative study of two groups set in the microsurgical skills lab. One resident, who never practiced microsurgery, and one experienced microsurgeon were enrolled to perform anastomosis of chicken femoral vessels, bought from a supermarket, using a smartphone camera and a surgical microscope. Forty anastomoses of non-living chicken femoral vessels were divided into four groups. An experienced microsurgeon and a resident performed microsurgical anastomoses on femoral arteries from chicken thighs, five times with 8-0 nylon and five times with $10-0$ nylon, with both a smartphone and surgical microscope. The practitioners each completed one anastomosis per day to avoid the fatigue effect. Fig. 1 demonstrates the view from the smartphone screen.

The smartphone surgery was performed using a Samsung Galaxy S9 plus smartphone under $\times 10$ magnification. The smartphone was placed on a stand, approximately $10 \mathrm{~cm}$ from the vessel, and a light source was provided by the camera's LED light as shown in Fig. 2. The magnification of the image on the
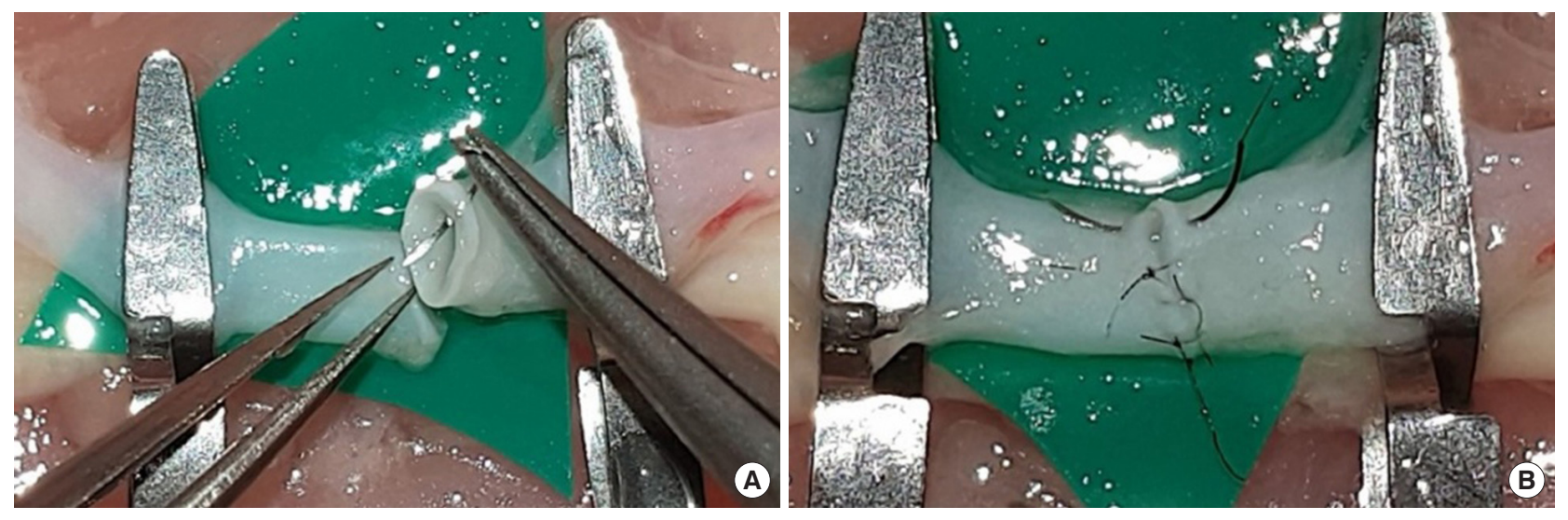

Fig. 1. The view from the digital screen of the smartphone while performing the practice under $\times 10$ magnification $(A, B)$. 
screen depended on the distance between the vessel and the smartphone; commercially available smartphone stands are usually shorter than the distance between the lens of an operative microscope and the vessel. The shorter the distance between lens and object, the higher the magnification. In this study, the $\times 10$ magnification image on a smartphone was equal to the image on a $\times 20$ magnification operative microscope. The microscope used in this study was a Leica M525 MS3 surgical microscope (Wetzlar, Germany) with $\times 16-20$ magnification.

The time taken to perform each anastomosis and the number of errors according to the anastomosis lapse index (ALI) [8] were recorded. The ALI describes the characteristics of ten suturing errors in anastomosis: (1) disruption of the anastomosis line (direct visualization and leakage of ink through the anasto-

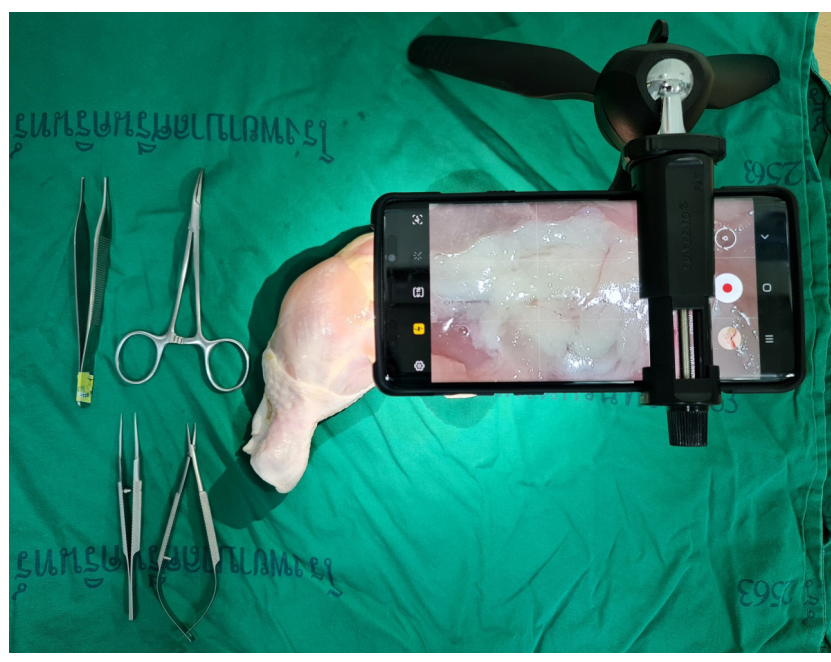

Fig. 2. Set-up of the operation under a smartphone. mosis); (2) the suture catching the backwall or sidewall of the vessel; (3) placement of an oblique stitch causing tissue distortion; (4) unusually large bites causing tissue enfoldment; (5) partial-thickness stitch (does not go through the full thickness of the vessel); (6) unequal distancing of sutures; (7) visible tear in vessel wall; (8) tight sutures causing strangulation of edges; (9) thread in the lumen; and (10) internal valve or large edge overlap. The ALI was assessed under an operative microscope by an author who was blinded to the variables in the study.

The normality of the data was tested by the Shapiro-Wilk test. The Student t-test was used for statistical analysis for normally distributed data, and the Mann-Whitney U test was used for non-normally distributed data. Results were considered statistically significant at $\mathrm{P}<0.05$.

\section{RESULTS}

\section{Time taken to perform anastomosis}

For the experienced microsurgeon, the median operation time when using the smartphone was 32.5 minutes; this was significantly longer than the mean operation time of 20 minutes when using the microscope (Table 1). In subgroup analysis, the only significant difference in the mean operation time for the experienced microsurgeon was between using the smartphone and the microscope with $8-0$ sutures $(\mathrm{P}=0.021)$. A subgroup analysis of the resident showed no significant difference in mean operation times between the smartphone and the microscope groups (Table 2). The operation time while using a smartphone by the resident was not significantly different when compared to the experienced microsurgeon $(\mathrm{P}=0.238)$. In contrast, the

Table 1. Comparison of the performance of an experienced microsurgeon using a smartphone versus an operative microscope for anastomosis in a non-living chicken vessel

\begin{tabular}{lcccc}
\hline Experienced microsurgeon & No. of anastomoses & Smartphone, median (range) & Microscope, median (range) & P-value \\
\hline Time (min) & 10 & $32.5(23-47)$ & $20.0(14-34)$ & 0.001 \\
8-0 suture & 5 & $35.0(23-41)$ & $20.0(14-25)$ & 0.021 \\
10-0 suture & 5 & $27.0(27-47)$ & $22.0(19-34)$ & 0.090 \\
Anastomosis lapse index errors & 10 & $1.0(0-3)$ & $0.0(0-2)$ & 0.485 \\
8-0 suture & 5 & $0.0(0-3)$ & $0.0(0-1)$ & 0.519 \\
10-0 suture & 5 & $1.0(0-2)$ & $1.0(0-2)$ & 1.000 \\
\hline
\end{tabular}

Table 2. Comparison of the performance of a resident using a smartphone versus an operative microscope for anastomosis in a non-living chicken vessel

\begin{tabular}{lcccc}
\hline Resident & No. of anastomoses & Smartphone, median (range) & Microscope, median (range) & P-value \\
\hline Time $(\mathrm{min})$ & 10 & $35.5(27-48)$ & $35.0(30-45)$ & 1.000 \\
8-0 suture & 5 & $36.0(32-42)$ & $41.0(35-45)$ & 0.523 \\
10-0 suture & 5 & $35.0(27-48)$ & $35.0(30-38)$ & 0.831 \\
Anastomosis lapse index errors & 10 & $4.0(0-7)$ & $1.5(0-5)$ & 0.065 \\
8-0 suture & 5 & $4.0(0-5)$ & $1.0(1-5)$ & 0.390 \\
10-0 suture & 5 & $4.0(1-7)$ & $2.0(0-3)$ & 0.205 \\
\hline
\end{tabular}


operation time of the experienced microsurgeon was significantly shorter than the resident when using the operative microscope $(\mathrm{P}<0.001)$.

\section{Number of ALI errors}

Samples of anastomosis and ALI error scores from the smartphone group are shown in Fig. 3. For the resident, there was no significant difference in the number of ALI errors between the smartphone and microscope groups. No statistical difference in error scores was detected among the anastomoses of the experienced surgeon. The experienced surgeon had better ALI error scores than the resident in both the smartphone and operative microscope groups $(\mathrm{P}=0.006$ and $\mathrm{P}=0.025$, respectively). The two most common errors in the smartphone group, by the resident, were partial-thickness stitches and anastomosis line disruption. When using the smartphone, the experienced microsurgeon made four partial-thickness errors, while this error occurred in 8 of 10 anastomoses performed by the resident. In

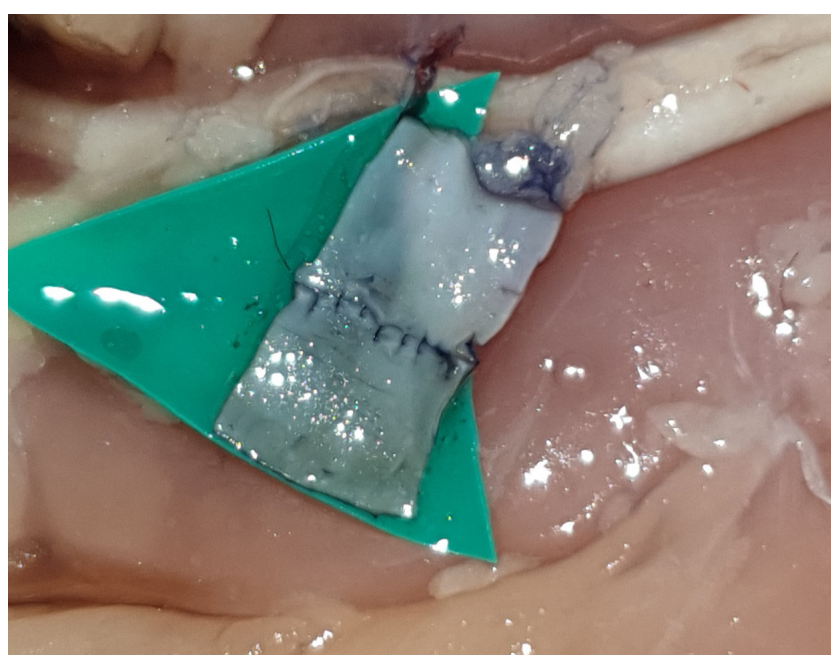

Fig. 3. The anastomosis of a vessel was evaluated using the anastomosis lapse index. contrast, in the microscope group, the resident made only one partial-thickness error. When using the smartphone, anastomosis line disruption was detected in one anastomosis performed by the experienced surgeon and eight anastomoses by the resident. In the microscope group, there was one anastomosis line disruption error by the experienced surgeon, and three errors by the resident. The errors are described in detail in Table 3.

\section{DISCUSSION}

Microsurgery with an on-screen microscope has been advocated for decades. Franken et al. [9] reported the three-dimensional on-screen microsurgical system and described that this system increased surgeons' comfort level, but required further refinement. Later, there were several reports highlighting the promising results of endoscopic- and laparoscopic-assisted systems for performing microsurgery [10-13]. A decade later, the video camera recorder was used as a microscope by de Barros et al. [14-17]. Currently, smartphone technology has dramatically improved, allowing many researchers to develop and try smartphone-assisted microsurgical systems $[2-7,18]$. Although, the results have been good in artificial models, living models such as the rat artery have seldom been successful. The current study demonstrates that the smartphone can potentially be used with the non-living chicken model for microsurgery skill training, since the mean number of ALI errors by the experienced microsurgeon showed no significant difference between the smartphone and microscope groups. There was a significantly longer operative time for the experienced microsurgeon using a smartphone, but the duration was not significantly different for the resident. Therefore, this study suggests a possible learning curve for experienced surgeons adjusting to the use of smartphones in microsurgery. The resident, who had never practiced the anastomosis microsurgery before this study, demonstrated no significant difference in mean operation time between groups. How-

Table 3. Anastomosis lapse index error scores using a smartphone versus an operative microscope

\begin{tabular}{|c|c|c|c|c|c|}
\hline \multirow{2}{*}{ Error } & \multicolumn{2}{|r|}{ Smartphone } & \multicolumn{2}{|c|}{ Operative microscope } & \multirow{2}{*}{ Total errors } \\
\hline & Resident & Experienced microsurgeon & Resident & Experienced microsurgeon & \\
\hline Disruption of the anastomosis line & 8 & 1 & 3 & 0 & 12 \\
\hline Partial-thickness stitch & 7 & 4 & 1 & 0 & 12 \\
\hline Unusually large bites causing tissue enfoldment & 3 & 2 & 5 & 2 & 12 \\
\hline Placement of an oblique stitch causing tissue distortion & 3 & 1 & 2 & 3 & 9 \\
\hline Tight sutures causing strangulation of edges & 4 & 1 & 3 & 0 & 8 \\
\hline Visible tear in vessel wall & 2 & 0 & 1 & 0 & 3 \\
\hline Thread in the lumen & 3 & 0 & 1 & 0 & 4 \\
\hline Unequal distancing of sutures & 3 & 0 & 1 & 0 & 4 \\
\hline Internal valve or large edge overlap & 3 & 0 & 0 & 1 & 4 \\
\hline The suture catching the backwall or sidewall of the vessel & 0 & 0 & 2 & 0 & 2 \\
\hline Total errors by group & 36 & 9 & 19 & 6 & \\
\hline
\end{tabular}


ever, the ALI scores of the resident tended to be higher in the smartphone group, and reported frustration while using the smartphone. The conclusion of this study is that the smartphone is not suitable for a resident and may not replace standard surgical microscope practice, whereas an experienced surgeon could utilize the smartphone for microsurgery skill exercises at home or when resources are limited.

This study also identified previously reported disadvantages of using the smartphone, including the loss of binocular vision, or stereoscopic view, that made depth-of-field estimations difficult while working in three dimensions $[5,7]$. This was supported by the number of partial-thickness errors in this study. Moreover, the practitioners said that using magnification modes greater than $\times 4$ caused the image on the screen to become blurry. Initially, the available LED flashlight on the smartphone was used as a light source, but it was found that the quality of the picture improved substantially with the addition of a light lamp. However, even with extra light, practitioners reported that the digital image on a smartphone resulting from $\times 10$ zoom on a high-quality screen $(2,960 \times 1,440$ pixels $)$ could not replace the clear lens visualization of the surgical microscope. Therefore, smartphone microsurgery practice on small diameter vessels such as those in chicken wings or the femoral arteries of rats could prove more difficult $[6,7]$. The nylon $8-0$ and $10-0$ suture materials used in this study were approximately $50 \%$ of the total cost. To reduce this expense, some studies have demonstrated that human hair can be used as a replacement for nylon sutures in microsurgical training $[19,20]$.

The limitations of this study can be addressed in future studies. Only one resident and one experienced microsurgeon participated in this study. While the ALI scores represented errors in the performance of the anastomosis microsurgery, the difficulty of the procedure was not assessed and further study should explore technical aspects such as hand motion analysis as well as practitioner satisfaction scores. In this study, the ability of analysis to detect significant differences in some data and the subgroup analysis of suture materials may not have been sufficient. Though the diameters of the chicken femoral vessels were not measured for this study, others have reported variation in diameters $(1.68-3.25 \mathrm{~mm})[21,22]$.

In conclusion, using today's smartphone in microsurgical training with a non-living model was practical (available anywhere, anytime) and a valuable device for experienced practitioners who need to improve their microsurgical skills, especially when at home or when resources are limited. However, vessel anastomosis using a smartphone took longer to complete than with the surgical microscope and caused more errors when performed by a resident.

\section{NOTES}

\section{Conflict of interest}

No potential conflict of interest relevant to this article was reported.

\section{Ethical approval}

The study was approved by the Ethics Committee Khon Kaen University (approval No. HE631506) and performed in accordance with the principles of the Declaration of Helsinki. Written informed consent was obtained.

\section{Author contribution}

Conceptualization: S Jianmongkol, C Vinitpairot, S Wattanakamolchai. Data curation: S Jianmongkol, C Vinitpairot, N Thitiworakarn, S Wattanakamolchai. Formal analysis: S Jianmongkol, C Vinitpairot. Funding acquisition: S Jianmongkol, C Vinitpairot. Methodology: S Jianmongkol, C Vinitpairot, N Thitiworakarn, S Wattanakamolchai. Project administration: S Jianmongkol, C Vinitpairot, N Thitiworakarn, S Wattanakamolchai. Visualization: S Jianmongkol, C Vinitpairot, N Thitiworakarn, S Wattanakamolchai. Writing - original draft: S Jianmongkol. Writing - review \& editing: C Vinitpairot, $N$ Thitiworakarn, $S$ Wattanakamolchai.

\section{ORCID}

Surut Jianmongkol https://orcid.org/0000-0003-3554-4158 Chaiyos Vinitpairot https://orcid.org/0000-0002-6422-5095 Navapong Thitiworakarn

Settapon Wattanakamolchai https://orcid.org/0000-0003-3846-5885

https://orcid.org/0000-0001-7117-3142

\section{REFERENCES}

1. Mobasheri MH, Johnston M, Syed UM, et al. The uses of smartphones and tablet devices in surgery: a systematic review of the literature. Surgery 2015;158:1352-71.

2. Kim DM, Kang JW, Kim JK, et al. Microsurgery training using a smartphone. Microsurgery 2015;35:500-1.

3. Huotarinen A, Niemela M, Jahromi BR. Easy, efficient, and mobile way to train microsurgical skills during busy life of neurosurgical residency in resource-challenged environment. World Neurosurg 2017;107:358-61.

4. Malik MM, Hachach-Haram N, Tahir M, et al. Acquisition of basic microsurgery skills using home-based simulation training: a randomised control study. J Plast Reconstr Aesthet Surg 2017;70:478-86. 
5. Karakawa R, Yoshimatsu H, Nakatsukasa S, et al. A new method for microsurgery training using a smartphone and a laptop computer. Microsurgery 2018;38:124-5.

6. Ghabi A, Amar S, Harion M, et al. Microvascular anastomosis using loupes and smartphone magnification: experimental study for application to limited-resource environments. Hand Surg Rehabil 2020;39:92-5.

7. Teixeira RK, Feijo DH, Valente AL, et al. Can smartphones be used to perform video-assisted microanastomosis? An experimental study. Surg Innov 2019;26:371-5.

8. Ghanem AM, Al Omran Y, Shatta B, et al. Anastomosis lapse index (ALI): a validated end product assessment tool for simulation microsurgery training. J Reconstr Microsurg 2016;32:233-41.

9. Franken RJ, Gupta SC, Banis JC Jr, et al. Microsurgery without a microscope: laboratory evaluation of a three-dimensional on-screen microsurgery system. Microsurgery 1995; 16:746-51.

10. Southern SJ, Ramakrishnan V, Villofane O, et al. Video microsurgery: early experience with an alternative operating magnification system. Microsurgery 2001;21:63-9.

11. Gorman PJ, Mackay DR, Kutz RH, et al. Video microsurgery: evaluation of standard laparoscopic equipment for the practice of microsurgery. Plast Reconstr Surg 2001;108:8649.

12. El-Shazly M, El-Sonbaty M, Kamel A, et al. Endoscopic-assisted microsurgery: microsurgery in the new millennium? A comparative experimental study. Br J Plast Surg 2003;56: 37-40.

13. El-Shazly M, Chao HW, Cheng MH, et al. Advancement of the endoscopic assisted microsurgery: a clinical experience. Eur J Plast Surg 2005;28:272-5.

14. Barros RS, Brito MV, Moura GP, et al. Is it possible to do a microvascular anastomosis with an ordinary video camera? Experimental study. J Reconstr Microsurg 2011;27:503-8.

15. de Barros RS, Brito MV, de Brito MH, et al. Morphofunctional evaluation of end-to-side neurorrhaphy through video system magnification. J Surg Res 2018;221:64-8.

16. Monteiro de Barros RS, Brito MV, Teixeira RK, et al. Highdefinition video system for peripheral neurorrhaphy in rats. Surg Innov 2017;24:369-72.

17. Teixeira RK, Leal RA, Sabba MF, et al. Evaluation of 2 lowcost high-definition video systems for venous anastomosis. Surg Innov 2020;27:373-7.

18. Capkin S, Cavit A, Kaleli T. Microsurgery training with smartphone. Handchir Mikrochir Plast Chir 2018;50:4435.

19. Eroglu L, Guneren E, Akbas H, et al. Using human hair as suture material in microsurgical practice. J Reconstr Microsurg 2003; 19:37-40.

20. Luangjarmekorn P, Kitidumrongsook P, Honsawek S. Do-ityourself microsuture from human hair for basic microsurgical training. J Reconstr Microsurg 2019;35:315-21.

21. Kang BY, Jeon BJ, Lee KT, et al. Comprehensive analysis of chicken vessels as microvascular anastomosis training model. Arch Plast Surg 2017;44:12-8.

22. Szabo B, Fazekas L, Ghanem S, et al. Biomechanical comparison of microvascular anastomoses prepared by various suturing techniques. Injury 2020;51:2866-73. 